\title{
TIPOLOGÍA LEXICOGRÁFICA EN LA ENSEÑANZA DEL INGLÉS COMO SEGUNDA LENGUA: HACIA UNA DESCRIPCIÓN DEL USO DE LOS DICCIONARIOS
}

\author{
MARÍA DEL MAR SÁNCHEZ RAMOS \\ Universidad de Granada
}

\section{RESUMEN}

El presente artículo tiene como finalidad describir los principales diccionarios destinados a estudiantes de inglés como L2 y así contribuir a lo que se denomina tipología de diccionarios. Al mismo tiempo, el análisis refuerza la idea de que la tipología de diccionarios y las necesidades de los usuarios son dos conceptos íntimamente relacionados. A su vez, se exponen distintas cuestiones a la hora de abordar el uso del diccionario en las tareas productivas y receptivas y se incide en el hecho de que los estudiantes de una L2 necesitan un mayor entrenamiento en las destrezas relacionadas con el uso del diccionario para así beneficiarse de la información tan valiosa que estas herramientas de aprendizaje proporcionan.

\begin{abstract}
The current article aims to describe the main learners' dictionaries and therefore to contribute to what is called dictionary typology. In doing so, the analysis reinforces the idea that dictionary typology and user needs are concepts closely related. Thus, different perspectives are set out in order to deal with the use of dictionaries in both productive and receptive tasks and emphasis is placed on the fact that students of a L2 need more training into dictionary skills in order to take advantage of the valuable information dictionaries provide.
\end{abstract}

\section{INTRODUCCIÓN}

La llamada lexicografía pedagógica ha sido definida recientemente por R. Hartmann (2001: 26) como uno de los campos lingüísticos más prometedores cuya labor se encuentra a caballo entre la enseñanza y la elaboración de diccionarios. Fue en 1935 cuando esta disciplina inició su singladura partiendo del trabajo de dos lingüistas de renombre como 
M. West y J.G Endicott. Las primeras obras lexicográficas publicadas por destacados lingüistas como el mencionado $\mathrm{M}$. West evolucionaron y constituyeron, como se verá más adelante, el primer paso para el desarrollo de los distintos diccionarios que se conocen hoy en día, estableciéndose la tipología de diccionarios como disciplina dentro del campo de la investigación lexicográfica. Así, siguiendo esta línea, pretendo en este artículo ofrecer una aproximación teórica referida, por un lado, a la tipología de diccionarios y, por otro, al uso de los diccionarios en las tareas de producción y recepción. En primer lugar, centraré mi atención en desarrollar el marco teórico en el que emerge la lexicografía pedagógica tomando como punto de partida los trabajos de tres célebres lingüistas: H. Palmer, M. West y A. S. Hornby. A continuación, describiré tres tipos de diccionarios destinado a estudiantes de una L2: el diccionario monolingüe, el bilingüe y el llamado bilingualised dictionary. Finalmente, especificaré el uso de los diccionarios en las tareas de recepción y producción

\section{EL COMIENZO DE LA TRADICIÓN LEXICOGRÁFICA EN UNA L2: H. PALMER, M. WEST Y A.S. HORNBY}

Como señala R. Hartmann (1992: 151), la tradición lexicográfica de la lengua inglesa se remonta al siglo XVII con R. Cawdrey y su obra titulada Table Alphabeticall of Hard Words (1604). Este primer trabajo inició la producción de diversos diccionarios que parafraseaban palabras latinas en un inglés más sencillo y constituyó lo que pasaría a conocerse como the hard word tradition. Posteriormente, se comenzó a compilar palabras de todo tipo, no sólo aquellas que eran difícil de entender, apareciendo así otra variedad de libros de consulta: diccionarios generales, diccionarios especializados, etc.

En el caso de la enseñanza de una L2, ha habido una amplia producción de diccionarios a lo largo del siglo XX. Las figuras más relevantes y, en definitiva, los pioneros en este área de investigación fueron tres lingüistas consagrados que estaban a cargo de programas de investigación en la enseñanza de lengua extranjeras: H. E. Palmer, M. West. y A. S Hornby. Curiosamente estos tres estudiosos trabajaban en proyectos donde la producción de un diccionario para estudiantes no era precisamente la meta final.

El principal objetivo de H. Palmer era el de facilitar el proceso de aprendizaje de los alumnos de una L2 a través de un vocabulario limitado, es decir, se seleccionaban como unidades léxicas aquellas palabras que eran de mayor utilidad en la comunicación diaria. El concepto de vocabulario limitado es imprescindible cuando hablamos de obras de referencia destinadas a estudiantes de una L2 y hace alusión a la lista de palabras que un diccionario emplea en sus definiciones. Esta lista está formada por aquellas palabras que son de más utilidad en la comunicación diaria. En suma, la idea de utilizar un vocabulario limitado consiste en hacer que las definiciones de los diccionarios sean lo más claras y precisas posibles.

Este interés en la búsqueda de un vocabulario limitado también fue compartido por M. West, que por entonces trabajaba en la India compilando lecturas simplificadas para los escolares. $\mathrm{Su}$ investigación pretendía recoger un número de palabras indispensables en la enseñanza de una L2; y esto fue también el germen de los tradicionales recuentos y las listas de palabras . M. West y J.G. Endicott consiguieron fijar un vocabulario limitado de 
1.490 palabras para definir 23.898 unidades léxicas (Cowie, 1999:32) en su diccionario The New Method English Dictionary (1935). Esta obra ha sido de gran importancia en el campo lexicográfico, ya que asentó las bases para futuros recuentos léxicos que se utilizarían en las definiciones de las distintas entradas de un diccionario. De hecho, constituyó el origen del vocabulario controlado en la primera edición del diccionario monolingüe de Longman de 1978.

El primer recuento con fines pedagógicos se conoce como Thousand-word English (TWE), compilada por A. S. Hornby en 1937. Por un lado, las entradas eran palabras o lexemas, tal y como aparecen en los diccionarios y, a su vez, no se incluían ni las flexiones ni las formas derivadas de las palabras como entradas aparte, sino que aparecían como información adicional en la entrada.

\section{(1) druw $/ 2 \circ \rho / /$}

drew /dru/f pret.

drawn $d$ drL $\checkmark v$, past pp

(1.e.p., n picture)

(2.e-g., a line)

druwing $/ \mathrm{k}+\mathrm{N} / \mathrm{l}, \mathbf{n}$

A su vez, esta lista fue refinada por H. Palmer para la elaboración de su diccionario monolingüe, A Grammar of English Words (GEW, 1938). La estructura de las entradas era similar, aunque esta nueva publicación incluía ejemplos y frases idiomáticas:

(2) $\operatorname{sinft}$

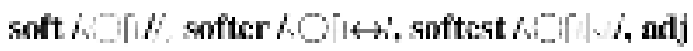

1. - not hard

a soft hed, soft leather [wood, etc.]

the ground is solt aller the ratin

[...]

suften...

Otra aportación al campo de la lexicografia pedagógica fue la de A.S. Hornby con la publicación en Japón de su diccionario Idiomatic and Syntactic English Dictionary (1942), conocido también como Advanced Learner's Dictionary (1948). En los años 70 otros diccionarios importantes han continuado esta tradición de los diccionarios pedagógicos: Longman Dictionary of Contemporary English, Chambers Universal Learner's Dictionary, etc. La década de los 80 estuvo marcada por el gran proyecto de J. Sinclair, The Cobuild Project, donde el trabajo lexicográfico fue substituido por la lingüística computacional y 
que culminaría con la edición de Collins English Dictonary, basado en una vasta selección de corpus orales informatizados. Finalmente, en los 90 podemos mencionar diccionarios de similares características: Cambridge International Dictionary of English, entre otros.

Como acabo de señalar en los anteriores párrafos, la producción de los diccionarios quedó establecida por los tres autores de renombre anteriormente mencionados, M. West (New Method Engish Dictionary, 1935), H. E. Palmer (A Grammar of English words, 1938) y A.S. Hornby (Idiomatic and Syntactic English Dictionary, 1942) y sus respectivos trabajos lexicográficos. Ellos propusieron una serie de principios que, con el paso del tiempo, adquirieron la categoría de convención a la hora de diseñar un diccionario monolingüe (Rundell, 1998:316):

1. Control del vocabulario: los tres autores coinciden, como ya ha sido apuntado, en el criterio de la selección de un vocabulario limitado. El objetivo es definir las palabras de forma clara y precisa, seleccionando para ello un número limitado de palabras para establecer las definiciones. Este hecho persigue solventar uno de los mayores problemas que los estudiantes apuntan en relación con el uso del diccionario: la dificultad a la hora de comprender las definiciones que un diccionario ofrece.

2. Información gramatical y sintáctica: las palabras no actúan aisladas, sino que se rigen en gran parte por comportamientos sintácticos específicos. Así, debido a que el uso del diccionario para los alumnos de una L2 atañe en ciertos casos las tareas productivas, las entradas deben mostrar información, entre otras, sobre la complementación verbal.

3. El papel de los ejemplos: como consecuencia de su carácter pedagógico, la inclusión de ejemplificación es una característica de máxima importancia, ya que clarifica en gran medida la definición de las palabras y hace más accesible la comprensión de las entradas.

4. Fraseología: el apartado de las frases léxicas constituye un punto importante en la enseñanza de lenguas. De este modo, es de máxima importancia que una obra destinada a estudiantes de L2 recoja aquellas unidades léxicas que aparecen en combinación más o menos fija y cuyos significados son diferentes de los que denotan sus elementos por separado.

\section{TIPOS DE DICCIONARIOS}

Una de las primeras decisiones que un estudiante de lenguas debe tomar es la elección de un buen diccionario. Los diccionarios monolingües y bilingües son los seleccionados con más frecuencia por los estudiantes, si bien existen otros más especializados que se hacen necesarios en los niveles superiores (tesauros, diccionarios de colocaciones, de frases idiomáticas, etc). No es posible reseñar el amplio abanico de obras lexicográficas destinadas a estudiantes de una L2 por motivos de espacio. De este modo, me centraré en aquellos que gozan de mayor popularidad entre el alumnado de una L2 (diccionarios 
monolingües y diccionarios bilingües) $\mathrm{y}$, aquellos que recientemente han irrumpido en el campo de la enseñanza de lenguas: los llamados bilingualised dictionaries.

\subsection{El diccionario monolingüe}

Los diccionarios monolingües están escritos en su totalidad en la L1. La entrada de la palabra, la definición, es decir, toda la información se encuentra en L1, en este caso el inglés. Esta es la principal ventaja que se suele aducir en favor de este tipo de diccionarios. No obstante, esta misma característica se señala como desventaja para áquellos que son partidarios del uso de diccionarios bilingües. Se advierte, en estos caso, que toda la amplia información que estos diccionarios aportan (información gramatical, definición, uso, frases hechas y colocaciones, ejemplos, etc) puede ser inútil si el alumno encuentra dificultades insoslayables en la inteligibilidad de abreviaturas, códigos gramaticales, etc.

El uso del diccionario monolingüe implica claramente un nivel determinado de competencia lingüística. P. Bogaards (1996), en su estudio comparativo sobre diccionarios, subraya que los diccionarios monolingües están generalmente indicados para alumnos de niveles superiores, ya que requieren un conocimiento más avanzado de la lengua, del vocabulario y de su metalenguaje.

Otro aspecto a considerar es el del vocabulario limitado que se emplea en las definiciones de los diccionarios monolingües. R. Allen (1996: 46) menciona que LDOCE fue el primero en utilizar un vocabulario limitado: unas 2000 palabras, y Cambridge International Dictionary of English-CIDE-y Collins COBUILD English Dictionary-COBUILD- usan prácticamente el mismo número. Por el contrario, OALD emplea en su última edición un vocabulario reducido a 3.500 palabras, que son listadas al final del diccionario.

\subsection{El diccionarios bilingüe}

El diccionario bilingüe emplea dos lenguas. Las entradas y los ejemplos están en L1 y sus correspondientes traducciones en L2. Los diccionarios bilingües han sido criticados por otra serie de razones. Se piensa que un uso no limitado de los diccionarios bilingües incrementa la tendencia de los estudiantes a traducir literalmente de L1; su uso también puede reforzar la creencia de que las palabras tienen siempre un único equivalente; no presentan un información sintáctica detallada, etc. (G. Thompson 1987). Contrariamente a los que rechazan el uso del diccionario bilingüe, R. Hartmann (1983: 198) realizó varios estudios para determinar la aceptación de estos diccionarios entre sus alumnos y concluyó que su uso estaba más extendido y, que dependiendo del tipo de tareas y de las necesidades de cada alumno, eran útiles en el aula de idiomas. Los resultados revelaban que sus alumnos, estudiantes ingleses de alemán, utilizaban el diccionario bilingüe para conocer el significado (97\%), la gramática (82\%), el uso (76\%), la ortografía (68\%), los sinónimos $(58 \%)$, la pronunciación $(15 \%)$, la etimología $(12 \%)$ y otros $(5 \%)$.

Los diccionarios bilingües también han mostrado ganancias léxicas en el campo de la adquisición de vocabulario (Bejoint 1987; Luppescu y Day 1993; Knight 1994). J. Hulstijn, M. Hollander y T. Greidanus (1996) resaltaron que una exposición repetida de palabras, 
con la ayuda de un glosario o de un diccionario bilingüe, aumentaba claramente el léxico en los alumnos de un nivel superior. Otro estudio digno de mención es el de S. Luppescu y R.R. Day (1993), en el que se trataba de examinar la correlación entre el uso de diccionario y la adquisición de vocabulario en la destreza lectora. Sus resultados indicaron que los diccionarios bilingües eran efectivos cuando la palabra no presentaba numerosas entradas, ya que en este caso podía inducir a error. También la investigación de S. Knight (1994) reiteró que el diccionario bilingüe era más efectivo en la destreza lectora que el monolingüe en alumnos de baja competencia lectora.

P. Nation (2000: 290) mantiene que un diccionario bilingüe, aunque no tiene toda la información de un monolingüe, es un recurso que puede utilizarse como complemento de este último: "they provide meaning in a very accesible way, and they can be bi-directionalEnglish-first language and first language-English".

\subsection{El bilingualised dictionary}

El último tipo de diccionario que voy a tratar es el conocido como bilingualised dictionary. Este diccionario ofrece la misma información que aparece en los monolingües además de la traducción de la entrada. Aunque su uso no es muy generalizado entre el alumnado español de una L2, creo que es conveniente incluirlo en este artículo como ejemplo de las recientes investigaciones lexicográficas. B. Laufer y L. Hadar (1997) evaluaron la eficacia de este diccionario frente al monolingüe y el bilingüe en las destrezas productivas y receptivas. Los resultados claramente demostraron la eficacia de este nuevo diccionario y concluyeron que "a hybrid dictionary that contains the two types of information (monolingual and bilingual) seems to be the most appropriate product of lexicographers' efforts" (Laufer y Hadar, 1997: 190). Estos datos finales dieron como resultado el lanzamiento de unos veinte bilingualised dictionaries en las últimas dos décadas, versiones de distintos diccionarios ingleses, siendo el primero el Oxford Student Dictionary for Hebrew Speakers (1978). En definitiva, el llamado bilingualised dictionary presentaba más beneficios que el bilingue en tareas de comprensión y también era significativamente superior que el monolingüe en las tareas de producción.

\section{USO DE LOS DICCIONARIOS EN LAS TAREAS PRODUCTIVAS Y RECEPTIVAS}

El diccionario, gracias a la ingente información que presenta, puede utilizarse en una amplia gama de actividades. Son dos las tareas para las que frecuentemente se emplea : tareas de producción y tareas de recepción. Teniendo en cuenta la división de las cuatro estrategias, la lectura y la comprensión auditiva se identifican con las tareas de recepción y las dos restantes, producción oral y escritura, con las de producción. Como puede apreciarse, el proceso que el alumno debe realizar en una u otra actividad es distinto y así el uso del diccionario será diferente dependiendo de cada tarea. H. Bejoint (1981:210) hace una amplia referencia a estos dos tipos de actividades : "the best dictionary for decoding is the one that contains the largest number of entries[...] the best dictionary for encoding is one that provides the most detailed guidance on syntax and collocation, including perhaps advice on pitfalls to avoid". 
P. Nation (2000), además de impulsar el uso del diccionario en las actividades de producción y recepción, manifiesta que el diccionario también puede utilizarse como un instrumento de aprendizaje. En su libro Learning vocabulary in another language describe de manera muy clara los principales fines para los que se utiliza el diccionario dependiendo de la actividad que se realice (Nation 2000: 281-282):

1. Comprehension (decoding)

- Look up unknown words met while listening, reading or translating.

- Confirm the meanings of partly known words.

- Confirm guesses from context.

2. Production (encoding)

- Look up unknown words needed to speak, write or translate.

- Look up the spelling, pronunciation, meaning, grammar, constrains on use, collocations, inflections and derived forms of partly known words needed to speak, write or translate.

- Confirm the spelling, pronunciation, meaning, etc. of known words.

- Check that a word exists.

- Find a different word to use instead of a known one.

- Correct an error.

3. Learning

- Choose unknown words to learn.

- Enrich knowledge of partly known words, including etymology.

\subsection{Uso del diccionario en tareas de producción}

En las actividades de producción el alumno intenta dar forma lingüística a una serie de ideas o conceptos, es decir, usa el lenguaje para transformar esas ideas en unidades léxicas. Sin embargo, es en este punto donde, según P. Scholfield (2000: 293), aparece la duda sobre la elección del tipo de diccionario: habrá que tener en cuenta el punto de partida, es decir, si la palabra es desconocida en parte o en su totalidad por el alumno:

In fact it is useful in relation to the productive use of reference words to deal separately with the two situations already mentioned- a) that where an L2 word is known for the meaning intended, but some aspect (s) of it are unknown/uncertain, and b) that where an L2 word is not known for the precise intended meaning at all.

De acuerdo con esta distinción podría explicarse el uso de uno otro tipo de diccionario. En el primer caso, aquél en que la palabra se conoce en la L2 sólo en parte, el uso del diccionario monolingüe puede ser efectivo, ya que se ayudará a aclarar aspectos como su uso, gramática, restricciones semánticas, etc. En este caso, el monolingüe ofrece mucha más información en comparación con el bilingüe, una información útil a la hora de escribir o hablar una L2. En segundo lugar, cuando se desconoce la palabra por completo en L2, el uso del diccionario monolingüe puede presentar grandes dificultades. Si no hay 
una palabra exacta en la L2 que buscar, la mejor opción es un diccionario bilingüe. Así, el papel del diccionario monolingüe constituye un segundo paso, una vez que el alumno se haya familiarizado de algún modo con la palabra. De este modo, el alumno puede consultar posteriormente el monolingüe para precisar el significado y obtener mayor información sobre la palabra.

Otro autor que se ha dedicado a indagar en este sector es M. Rundell, que también puntualiza la diferencia del uso del diccionario en las dos tareas (Rundell 1999: 36):

Each of these skills (receptive and productive) draws upon different areas of knowledge, and on the basis of tasks [...] it is possible to identify some of the types of information than students need access to in order to cope successfully with the sorts of productive operation at they are regularly called upon to perform .

El siguiente aspecto relacionado con las tareas de producción es el del tipo de información requerida del diccionario. La búsqueda del significado es una de las consultas más frecuentes en los diccionarios, pero es, a la vez, una de las que pueden conducir a más errores (Jain 1981; Fisher: 1994). M. P. Jain (1981) distinguió las dificultades que presentan los diccionarios monolingües en sus estructura u organización, y que en muchos casos eran fuente de errores para los alumnos en los ejercicios de producción. Esta misma autora añadió que, en algunos casos, las definiciones no eran suficientemente claras y precisas, y como consecuencia, pueden inducir a errores. Por ejemplo, la definición de collide en OALD (1980) es la siguiente: "to meet and strike together (usu. accidentally) with great force". En este caso el diccionario no muestra la información gramatical precisa: collide requiere un sujeto plural cuando no va seguido de la preposición "with". Esta falta de información llevaba a errores de producción como: it was a terrible sight: his car collided and he was thrown out of it. H. Nesi y P. Meara (1994: 7) también llevaron a cabo un estudio sobre los errores que aparecen en las tareas de producción. Como afirmaban en su trabajo, los resultados eran alarmantes: "the proportion of errors was very high [...] 402 $(56.5 \%)$ of all the sentences written after consulting a definition resulted in unacceptable use for the target words". Los errores que describieron eran muy variados: gramaticales, errores de combinación léxica, errores de tipo semántico, influencia de la técnica llamda Kridule ${ }^{1}$.

En esta línea, merece ser destacado el estudio llevado a cabo por G. Cumming, S. Cropp and R. Sussex (1994) que versaba sobre la manera de presentar las definiciones en el diccionario y su efecto en las tareas de producción. P. Nation (2000: 289) resumió los resultados de esta manera:

\footnotetext{
${ }^{1}$ Se trata de una estrategia usada por los alumnos cuando buscan una palabra en el diccionario. H. Nesi and P. Meara (1994:4) la describen así: kridule can be seen as a strategy which is made up of four separate steps: read the definition, select a short familiar segment, compose a sentence containing that segment ,substitute the target word for the selected segment. [...] this strategy can be weird and wonderful. Miller and Gildea cite such examples [...]: That news is very tenet, where the child appears to have substituted tenet for true, the last word in the entry :
}

tenet: opinión, belief, principle, or doctrine held as true. 
Cumming, Cropp and Sussex compared the effect of phrasal definition, sentence definition, phrasal definition with an example sentence, and sentence definitions with and example sentence. No difference was found on a production measure (write a sentence using the word ) and a comprehension measure (which of six sentences using the word are correct). Students indicated a clear preference for having example with definition and they favoured the sentence definition format.

Asimismo, el estudio realizado por B. Laufer (1993) ofrecía resultados similares. Tanto en las tares de comprensión como en las de producción, los alumnos encuestados preferían una definición combinada con el ejemplo.

P. Bogaards (1996) comenta que los principales problemas que los diccionarios presentan a la hora de llevar a cabo las tareas de producción son los relacionados con el acceso a las definiciones y con el uso correcto de las palabras. De manera muy breve, estos dos conceptos pueden ser resumidos así:

1. Acceso a las palabras : por un lado, en las tareas receptivas, el alumno debe ser guiado desde los elementos desconocidos a los familiares; por otro lado, en las productivas, el alumno debe comenzar por las palabras conocidas para así encontrar otras nuevas.

2. Uso correcto de las palabras : un uso adecuado de las palabras va más allá de las propiedades gramaticales, es decir, implica otros aspectos relacionados con las colocaciones y la pragmática entre otros.

Llegado este punto creo conveniente romper una lanza en favor de los diccionarios monolingües. No quiere esto decir que adopte la postura de aquellos que defiende al unísono los bilingües sino apostar por un uso más generalizado de la obra monolingüe en la enseñanza de una L2, sobre todo, en los estadios superiores del aprendizaje.

4.2 Uso del diccionario en las tareas de recepción

El uso del diccionario para fines receptivos supone la búsqueda de palabras en las tareas relacionados con dos de las cuatro habilidades en una L2: lectura y comprensión auditiva. Normalmente los estudios se centran en la primera de ellas (Bensoussan 1982; Laufer 1993; Luppescu y Day 1993; Knight 1994; Hulstjin, Hollander y Greidanus 1996; Scholfield 1999). No obstante, como apunta H. Bejoint (1981), el uso del diccionario también se extiende a las tareas de comprensión auditiva, aunque en menor medida. La principal razón a la hora de consultar el diccionario en relación con la destreza lectora se debe a la necesidad de conocer el significado. La búsqueda de palabras en el diccionario, en especial en el monolingüe, no es una tarea fácil si el alumno no está familiarizado con esta estrategia. P. Scholfield (1999) ha realizado un estudio muy interesante relacionado con este tema. Él propone cinco pasos que deben darse a la hora de utilizar el diccionario para fines receptivos:

a) Identificar la palabra desconocida.

b) Elegir la estrategia del uso del diccionario.

c) Encontrar la palabra en el diccionario.

d)Localizar la palabra y la información precisa.

e) Interpretar la entrada. 
Este mismo autor también menciona la importancia de determinar cuándo usar el diccionario. No siempre es la mejor opción cuando se busca el significado de palabras desconocidas, ya que no hay que olvidar que la estrategia de búsqueda de palabras en el diccionario supone una inversión de tiempo y puede entorpecer la concentración lectora (Luppescu y Day 1993). Por lo tanto, la primera cuestión será ver qué tipo de palabras son las que necesitan una búsqueda. En términos generales afirma que hay palabras que resultan familiares cuando se leen, pero que por el contrario necesitan una comprobación posterior (e.g. false friends). Otras son imprescindibles para la comprensión del texto (e.g. las que aparecen en el título, al principio del texto). Estas palabras claves facilitan la activación de las redes semánticas, permitiendo así una mayor comprensión del texto. Dependiendo también de la frecuencia con la que las palabras aparecen en un texto y áquellas que se repiten en los posteriores ejercicios sobre la lectura, la necesidad de su búsqueda será mayor o menor: una palabra que sea esencial en un texto y que aparezca de forma reiterada, necesitará antes o después un búsqueda precisa.

A pesar de la utilidad del diccionario en este tipo de tareas, los alumnos a veces encuentran dificultades cuando consultan este material. Como comenta P. Bogaards (1996:279), pueden presentarse dos tipos de problemas cuando se buscan palabras para las actividades de recepción: el primer problema se relaciona con la capacidad de encontrar la palabra; el segundo, con la capacidad de comprender de una manera apropiada la unidad léxica. Si el alumno no está entrenado, le será difícil encontrar la palabra que busca. Asimismo, no todos los diccionarios muestran la misma organización, y el aprendiz puede encontrarse con la complejidad en las entradas y subentradas que pueden inducirle a error. En cuanto al problema del significado de las unidades léxicas, debe tenerse en cuenta que ya muchos diccionarios han seguido la línea presentada por Longman de utilizar un vocabulario limitado que no dificulte el entendimiento del significado. Al igual que Longman, los cuatro diccionarios que compara P. Bogaards en su artículo (COBUILD, LDOCE, OALD, CIDE) ofrecen un vocabulario limitado de unas 2000 palabras aproximadamente. Otros recursos también se utilizan para la mejor comprensión de las unidades léxicas, como las ilustraciones, los ejemplos, notas de uso, etc.

En definitiva, de todo lo expuesto en este último apartado se puede deducir que un buen uso del diccionario reportaría indudables beneficios a los estudiantes de una L2. Como he comentado anteriormente, son varios los problemas que encuentran los alumnos a la hora de utilizar un diccionario, pero cabría cuestionarse si esta dificultad radica en los propios diccionarios o en la falta de entrenamiento por parte de los estudiantes. Naturalmente, no se trata de decidir si se trata de uno u otro motivo, pero sí considero importante cuestionarse hasta qué punto nuestros alumnos son usuarios eficientes de diccionarios.

\section{CONCLUSIÓN}

A modo de conclusión, podemos afirmar que se hace imprescindible un conocimiento y un estudio de la tipología de diccionarios para así orientar a los estudiantes de una L2 atendiendo a sus necesidades. De este modo, se ha pretendido establecer una descripción detallada de tres tipos de diccionarios pedagógicos. Otro aspecto que se ha considerado de 
máxima importancia y, a su vez, relacionado con la tipología lexicográfica, es el uso de los diccionarios. Así, se han expuesto las principales cuestiones a la hora de usar el diccionario en las tareas de producción y recepción tales como la elección del tipo de diccionario, información más buscada, dificultades en la consulta de entradas, etc.

En suma, la intención de este artículo ha sido mostrar en detalle tres de los diccionarios destinados a aprendices de una L2 teniendo en cuenta sus necesidades y destacar el uso de los diccionarios en las tareas de recepción y producción.

\section{REFERENCIAS BIBLIOGRÁFICAS}

Allen, R. 1996. “The Big Four”. English Today 46, 12/2: 41-47.

BEJoInt, H. 1981. "The foreign student's use of monolingual English dictionaries: A study language needs and reference skills". Applied Linguistics 2, 3: 207-221.

.y Moulin, A. 1987. "The place of the dictionary in an EFL programme". Ed. A. P. Cowie . Tübingen: M. Niemeyer. 97-104.

Bensoussan, M. 1983. "Dictionaries and tests of EFL reading comprehension". ELT Journal 37, 4: 341-345.

BogaARds, P. (1996). “ Dictionaries for learners of English”. International Journal of Lexicography 9, 4: 276-317.

Cowie, A (ed). 1987. The dictionary and the Language Learner. Lexicographica Series Maior 17. Tübingen: M. Niemeyer.

. 1999. English Dictionaries for Foreign Learners - A History. Oxford: Clarendom Press.

Cumming. G, Cropp. S, Sussex, R. 1994. “On line lexical resources for language learners: assessment of some approaches to word definitions". System 22, 3: 369-377.

FISHER, U. 1994. "Learning words from context and dictionaries: An experimental comparison". Applied Psycholinguistics 15: 551-574.

HaRtmann, R.R.K. (1983). “ The bilingual learner's dictionary and its users”. Multilingua 2, 4: 195-201.

.1992."Lexicography, with particular reference to English learners dictionaries". Language Teaching 25, 3: 151-159. .2001. Teaching and researching lexicography. Essex: Pearson Education

Hulstijn,J; Hollander, M \& Greidanus,T. 1996. "Incidental vocabulary learning by advanced foreign language students: the influence of marginal glosses, dictionary use, and reoccurrence of unknown words". The Modern Language Journal 80: 327-339.

JAIN, M.P. 1981. "On meaning in the foreign learner's dictionary", Applied Linguistics 2, 3: 274-286. 
KNIGHT, S. 1994. "Dictionary use while reading. The effects on comprehension and vocabulary acquisition for students of different verbal abilities". The Modern Language Journal 78, 3: 285-299.

LAUFER, B. 1993. "The effect of dictionary definitions and examples on the use as comprehension on new L2 words". Cahiers de Lexicology 63: 131-142.

.y HADAR,L. 1997. "Assessing the effectiveness of monolingual, bilingual, and "bilingualised" dictionaries in the comprehension and production of new words". The Modern Language Journal 81: 189-196.

Luppescu, S y DAY, R.R. 1993. "Reading, Dictionaries and Vocabulary Learning". Language Learning 43, 2: 263-287.

NATION P. 2000. Learning vocabulary in another language. New York: Cambridge University Press

Nesi, H y Meara, P. 1994. "Patterns of misinterpretation in the productive use of EFL dictionary definitions". System 22, 1: 1-15.

Nesi, H. 2000. The Use and Abuse of EFL Dictionaries. Lexicographica Series Maior 98, Tübingen: Max Niemeyer Verlag.

RUNDELL, M. 1998. "Recent trends in English pedagogical lexicography”. International Journal of Lexicography 11, 4: 315-342.

.1999. "Dictionary use in production". International journal of lexicography 12, 1: 35-53.

Schmitt, N y. McCARthy, M. 2000. Vocabulary acquisition and pedagogy. Cambridge: Cambridge University Press.

Schoffield, P. 1999. "Dictionary use in reception". International Journal of lexicography 12, 1: 13-34.

. 2000. "Vocabulary reference works in foreign language learning" Ed.

N. Schmitt y M. McCarthy . Cambridge: Cambridge university Press: 279-302.

Thompson, G. 1987. “Using bilingual dictionaries”. ELT Journal 41, 4: 282-286. 